\title{
Refined quadratic estimations of Shafer's inequality
}

\section{Yusuke Nishizawa*}

\section{"Correspondence: yusuke@ube-k.ac.jp General Education, Ube National College of Technology, Tokiwadai 2-14-1, Ube, Yamaguchi 755-8555, Japan}

\section{Abstract}

We establish an inequality by quadratic estimations; the double inequality

$$
\frac{\pi^{2} x}{4+\sqrt{\left(\pi^{2}-4\right)^{2}+(2 \pi x)^{2}}}<\arctan x<\frac{\pi^{2} x}{4+\sqrt{32+(2 \pi x)^{2}}}
$$

holds for $x>0$, where the constants $\left(\pi^{2}-4\right)^{2}$ and 32 are the best possible.

MSC: Primary 26D15;42A10

Keywords: Shafer's inequality; an upper bound for arctangent; a lower bound for arctangent

\section{Introduction}

Shafer [1-3] showed that the inequality

$$
\arctan x>\frac{8 x}{3+\sqrt{25+\frac{80}{3} x^{2}}}
$$

holds for $x>0$. Various Shafer-type inequalities are known, and they have been applied, extended and refined, see [4-8] and [9-12]. Especially, Zhu [12] showed an upper bound for inequality (1.1) and proved that the following double inequality

$$
\frac{8 x}{3+\sqrt{25+\frac{80}{3} x^{2}}}<\arctan x<\frac{8 x}{3+\sqrt{25+\frac{256}{\pi^{2}} x^{2}}}
$$

holds for $x>0$, where the constants $80 / 3$ and $256 / \pi^{2}$ are the best possible. Recently, in [8], Sun and Chen proved that the following inequality

$$
\arctan x<\frac{8 x+\frac{32}{4725} x^{7}}{3+\sqrt{25+\frac{80}{3} x^{2}}}
$$

holds for $x>0$; moreover, they showed that the inequality

$$
\frac{8 x+\frac{32}{4725} x^{7}}{3+\sqrt{25+\frac{80}{3} x^{2}}}<\frac{8 x}{3+\sqrt{25+\frac{256}{\pi^{2}} x^{2}}}
$$

\section{Springer}


holds for $0<x<x_{0} \cong 1.4243$. In this paper, we shall establish the refinements of inequalities (1.2) and (1.3).

\section{Results and discussion}

Motivated by (1.2), (1.3) and (1.4), in this paper, we give inequalities involving arctangent. The following are our main results.

Theorem 2.1 For $x>0$, we have

$$
\frac{\pi^{2} x}{4+\sqrt{\left(\pi^{2}-4\right)^{2}+(2 \pi x)^{2}}}<\arctan x<\frac{\pi^{2} x}{4+\sqrt{32+(2 \pi x)^{2}}}
$$

where the constants $\left(\pi^{2}-4\right)^{2}$ and 32 are the best possible.

Theorem 2.2 For $x>\alpha$, we have

$$
\frac{\pi^{2} x}{4+\sqrt{\left(\pi^{2}-4\right)^{2}+(2 \pi x)^{2}}}>\frac{8 x}{3+\sqrt{25+\frac{80}{3} x^{2}}}
$$

where the constant $\alpha=\sqrt{\frac{9600-1860 \pi^{2}+90 \pi^{4}}{2304-480 \pi^{2}+25 \pi^{4}}} \cong 2.26883$ is the best possible.

Theorem 2.3 For $x>\beta$, we have

$$
\frac{8 x}{3+\sqrt{25+\frac{256}{\pi^{2}} x^{2}}}>\frac{\pi^{2} x}{4+\sqrt{32+(2 \pi x)^{2}}}
$$

where the constant $\beta=\sqrt{\frac{4096+1536 \pi^{2}-528 \pi^{4}+24 \pi^{6}+\pi^{8}}{4096 \pi^{2}-768 \pi^{4}+36 \pi^{6}}} \cong 1.30697$ is the best possible.

Theorem 2.4 For $x>\gamma$, we have

$$
\frac{8 x+\frac{32}{4725} x^{7}}{3+\sqrt{25+\frac{80}{3} x^{2}}}>\frac{\pi^{2} x}{4+\sqrt{32+(2 \pi x)^{2}}},
$$

where the constant $\gamma \cong 1.38918$ is the best possible and satisfies the equation

$$
\begin{gathered}
151200-14175 \pi^{2}+128 \gamma^{6}-1575 \sqrt{15} \pi^{2} \sqrt{15+16 \gamma^{2}} \\
+75600 \sqrt{8+\pi^{2} \gamma^{2}}+64 \gamma^{6} \sqrt{8+\pi^{2} \gamma^{2}}=0 .
\end{gathered}
$$

From Theorems 2.1, 2.2, 2.3 and 2.4, we can get the following proposition, immediately.

Proposition 2.5 The double inequality (2.1) is sharper than (1.2) for $x>\alpha$. Moreover, the right-hand side of (2.1) is sharper than (1.3) for $x>\gamma$. 


\subsection{Proof of Theorem 2.1}

Becker-Stark's inequality is known as the inequality

$$
\frac{8}{\pi^{2}-4 x^{2}}<\frac{\tan x}{x}<\frac{\pi^{2}}{\pi^{2}-4 x^{2}}
$$

which holds for $0<x<\pi / 2$. Also, Becker-Stark's inequality (2.5) has various applications, extensions and refinements, see [13-16] and [17-19]. Especially, Zhu [19] gave the following refinement of (2.5): The inequality

$$
\frac{8}{\pi^{2}-4 x^{2}}+\frac{2}{\pi^{2}}-\lambda\left(\pi^{2}-4 x^{2}\right)<\frac{\tan x}{x}<\frac{8}{\pi^{2}-4 x^{2}}+\frac{2}{\pi^{2}}-\mu\left(\pi^{2}-4 x^{2}\right)
$$

holds for $0<x<\pi / 2$, where the constants $\lambda=\left(\pi^{2}-9\right) /\left(6 \pi^{4}\right)$ and $\mu=\left(10-\pi^{2}\right) / \pi^{4}$ are the best possible. In this paper, the result of Zhu (2.6) plays an important role in the proof of Theorem 2.1.

Proof of Theorem 2.1 The equation

$$
\arctan x=\frac{\pi^{2} x}{4+\sqrt{c+(2 \pi x)^{2}}}
$$

is equivalent to

$$
c=\frac{\pi^{4} x^{2}-8 \pi^{2} x \arctan x+16 \arctan ^{2} x-4 \pi^{2} x^{2} \arctan ^{2} x}{\arctan ^{2} x} .
$$

We set $t=\arctan x$, then

$$
\begin{aligned}
c & =\frac{\pi^{4} \tan ^{2} t}{t^{2}}-\frac{8 \pi^{2} \tan t}{t}+16-4 \pi^{2} \tan ^{2} t \\
& =16+F_{1}(t) .
\end{aligned}
$$

First, we assume that $0<t \leq 1 / 2$. Here, the derivative of $F_{1}(t)$ is

$$
\begin{aligned}
F_{1}^{\prime}(t) & =-\frac{8 \pi^{2} \sec ^{2} t}{t}+\frac{8 \pi^{2} \tan t}{t^{2}}-8 \pi^{2} \sec ^{2} t \tan t+\frac{2 \pi^{4} \sec ^{2} t \tan t}{t^{2}}-\frac{2 \pi^{4} \tan ^{2} t}{t^{3}} \\
& =\frac{\sin t}{\cos ^{2} t}\left(-\frac{8 \pi^{2}}{t \sin t}+\frac{8 \pi^{2} \cos t}{t^{2}}-\frac{8 \pi^{2}}{\cos t}+\frac{2 \pi^{4}}{t^{2} \cos t}-\frac{2 \pi^{4} \sin t}{t^{3}}\right) \\
& =\frac{\sin t}{\cos ^{2} t} F_{2}(t) .
\end{aligned}
$$

Since we have

$$
t-\frac{t^{3}}{6}<\sin t<t-\frac{t^{3}}{6}+\frac{t^{5}}{120}
$$

and

$$
1-\frac{t^{2}}{2}+\frac{t^{4}}{24}-\frac{t^{6}}{720}<\cos t<1-\frac{t^{2}}{2}+\frac{t^{4}}{24}
$$


for $0<t<\pi / 2$, the following inequality holds:

$$
\begin{aligned}
F_{2}(t)< & -\frac{8 \pi^{2}}{t\left(t-\frac{t^{3}}{6}+\frac{t^{5}}{120}\right)}+\frac{8 \pi^{2}\left(1-\frac{t^{2}}{2}+\frac{t^{4}}{24}\right)}{t^{2}} \\
& -\frac{8 \pi^{2}}{\left(1-\frac{t^{2}}{2}+\frac{t^{4}}{24}\right)}+\frac{2 \pi^{4}}{t^{2}\left(1-\frac{t^{2}}{2}+\frac{t^{4}}{24}-\frac{t^{6}}{720}\right)}-\frac{2 \pi^{4}\left(t-\frac{t^{3}}{6}\right)}{t^{3}} \\
= & \frac{\pi^{2} F_{3}(t)}{3\left(120-20 t^{2}+t^{4}\right)\left(24-12 t^{2}+t^{4}\right)\left(-720+360 t^{2}-30 t^{4}+t^{6}\right)},
\end{aligned}
$$

where $F_{3}(t)=82944000-8294400 \pi^{2}-72990720 t^{2}+7084800 \pi^{2} t^{2}+24883200 t^{4}-$ $2246400 \pi^{2} t^{4}-4832640 t^{6}+371520 \pi^{2} t^{6}+596736 t^{8}-35904 \pi^{2} t^{8}-48192 t^{10}+2076 \pi^{2} t^{10}+$ $2472 t^{12}-68 \pi^{2} t^{12}-74 t^{14}+\pi^{2} t^{14}+t^{16}$. We set $s=t^{2}$, then

$$
\begin{aligned}
F_{3}(t)> & 82944000-8294400\left(\frac{315}{100}\right)^{2}-72990720 s+7084800\left(\frac{314}{100}\right)^{2} s \\
& +24883200 s^{2}-2246400\left(\frac{315}{100}\right)^{2} s^{2}-4832640 s^{3}+371520\left(\frac{314}{100}\right)^{2} s^{3} \\
& +596736 s^{4}-35904\left(\frac{315}{100}\right)^{2} s^{4}-48192 s^{5}+2076\left(\frac{314}{100}\right)^{2} s^{5} \\
& +2472 s^{6}-68\left(\frac{315}{100}\right)^{2} s^{6}-74 s^{7}+\left(\frac{314}{100}\right)^{2} s^{7}+s^{8} \\
= & 642816-\frac{78435648 s}{25}+2593296 s^{2}-\frac{146200176 s^{3}}{125}+\frac{6011964 s^{4}}{25} \\
& -\frac{17327169 s^{5}}{625}+\frac{179727 s^{6}}{100}-\frac{160351 s^{7}}{2500}+s^{8} \\
= & \frac{1}{2500}\left(1607040000-7843564800 s+6483240000 s^{2}-2924003520 s^{3}\right. \\
& \left.+601196400 s^{4}-69308676 s^{5}+4493175 s^{6}-160351 s^{7}+2500 s^{8}\right) \\
= & \frac{1}{2500}\left(1607040000-7843564800 s+\left(\frac{7}{8}\right) 6483240000 s^{2}\right. \\
& +s^{2}\left(\left(\frac{1}{8}\right) 6483240000-2924003520 s+601196400 s^{2}-69308676 s^{3}\right) \\
& \left.+s^{6}\left(4493175-160351 s+2500 s^{2}\right)\right) \\
& \frac{1}{2500}\left(F_{4}(s)+s^{2} F_{5}(s)+s^{6} F_{6}(s)\right) .
\end{aligned}
$$

We shall show that the functions $F_{4}(s)>0, F_{5}(s)>0$ and $F_{6}(s)>0$. Here,

$$
\begin{aligned}
F_{4}(s) & =5400\left(297600-1452512 s+1050525 s^{2}\right) \\
& =5400 F_{7}(t) .
\end{aligned}
$$


The derivative of $F_{7}(t)$ is

$$
\begin{aligned}
F_{7}^{\prime}(s) & =2(-726256+1050525 s) \\
& \leq 2\left(-726256+1050525\left(\frac{1}{4}\right)\right) \\
& =-\frac{1854499}{2} .
\end{aligned}
$$

Since $F_{7}(s)$ is strictly decreasing for $0<s<1 / 4$ and $F_{7}(1 / 4)=2077 / 16$, we have $F_{4}(s)>0$.

$$
\begin{aligned}
F_{5}(s) & =36\left(22511250-81222320 s+16699900 s^{2}-1925241 s^{3}\right) \\
& >36\left(22511250-81222320 s-1925241 s^{3}\right) \\
& \geq 36\left(22511250-81222320\left(\frac{1}{4}\right)-1925241\left(\frac{1}{4}\right)^{3}\right) \\
& =\frac{1854335151}{16}
\end{aligned}
$$

and

$$
\begin{aligned}
F_{6}(s) & >4493175-160351\left(\frac{1}{4}\right) \\
& =\frac{17812349}{4} .
\end{aligned}
$$

Therefore, we can get $F_{3}(t)>0$. By $120-20 t^{2}+t^{4}>0,24-12 t^{2}+t^{4}>0$ and $-720+$ $360 t^{2}-30 t^{4}+t^{6}<0$, thus $F_{2}(t)<0$ and $F_{1}(t)$ is strictly decreasing for $0<t<1 / 2$. From $F_{1}(0+)=\left(\pi^{2}-4\right)^{2}-16$, we can get

$$
F_{1}\left(\frac{1}{2}\right) \leq F_{1}(t)<\left(\pi^{2}-4\right)^{2}-16
$$

for $0<t \leq 1 / 2$. Next, we assume that $1 / 2<t<\pi / 2$. From inequality (2.6), we have

$$
\begin{aligned}
- & 8 \pi^{2}\left\{\frac{2}{\pi^{2}}+\frac{8}{\pi^{2}-4 t^{2}}-\frac{\left(10-\pi^{2}\right)\left(\pi^{2}-4 t^{2}\right)}{\pi^{4}}\right\} \\
& +\pi^{2}(\pi-2 t)(\pi+2 t)\left\{\frac{2}{\pi^{2}}+\frac{8}{\pi^{2}-4 t^{2}}-\frac{\left(-9+\pi^{2}\right)\left(\pi^{2}-4 t^{2}\right)}{6 \pi^{4}}\right\}^{2} \\
< & F_{1}(t) \\
< & -8 \pi^{2}\left\{\frac{2}{\pi^{2}}+\frac{8}{\pi^{2}-4 t^{2}}-\frac{\left(-9+\pi^{2}\right)\left(\pi^{2}-4 t^{2}\right)}{6 \pi^{4}}\right\} \\
& +\pi^{2}(\pi-2 t)(\pi+2 t)\left\{\frac{2}{\pi^{2}}+\frac{8}{\pi^{2}-4 t^{2}}-\frac{\left(10-\pi^{2}\right)\left(\pi^{2}-4 t^{2}\right)}{\pi^{4}}\right\}^{2}
\end{aligned}
$$

and

$$
\frac{G_{1}(t)}{36 \pi^{6}}<F_{1}(t)<\frac{G_{2}(t)}{3 \pi^{6}},
$$


where

$$
\begin{aligned}
G_{1}(t)= & 4761 \pi^{6}-426 \pi^{8}+\pi^{10}-18252 \pi^{4} t^{2}+1944 \pi^{6} t^{2}-12 \pi^{8} t^{2} \\
& +7344 \pi^{2} t^{4}-1248 \pi^{4} t^{4}+48 \pi^{6} t^{4}-5184 t^{6}+1152 \pi^{2} t^{6}-64 \pi^{4} t^{6}
\end{aligned}
$$

and

$$
\begin{aligned}
G_{2}(t)= & -276 \pi^{6}+4 \pi^{8}+3 \pi^{10}-624 \pi^{4} t^{2}+416 \pi^{6} t^{2}-36 \pi^{8} t^{2} \\
& +12480 \pi^{2} t^{4}-2688 \pi^{4} t^{4}+144 \pi^{6} t^{4}-19200 t^{6}+3840 \pi^{2} t^{6}-192 \pi^{4} t^{6} .
\end{aligned}
$$

We set $s=t^{2}$, then

$$
\begin{aligned}
G_{1}(t)= & 4761 \pi^{6}-426 \pi^{8}+\pi^{10}-12 \pi^{4}\left(1521-162 \pi^{2}+\pi^{4}\right) s \\
& +48(-3+\pi) \pi^{2}(3+\pi)\left(-17+\pi^{2}\right) s^{2}-64(-3+\pi)^{2}(3+\pi)^{2} s^{3} \\
= & G_{3}(s)
\end{aligned}
$$

and

$$
\begin{aligned}
G_{2}(t)= & -276 \pi^{6}+4 \pi^{8}+3 \pi^{10}-4 \pi^{4}\left(156-104 \pi^{2}+9 \pi^{4}\right) s \\
& +48 \pi^{2}\left(\pi^{2}-10\right)\left(-26+3 \pi^{2}\right) s^{2}-192\left(\pi^{2}-10\right)^{2} s^{3} \\
= & G_{4}(s) .
\end{aligned}
$$

The derivatives of $G_{3}(s)$ are

$$
\begin{aligned}
G_{3}^{\prime}(s)= & 12\left(-1521 \pi^{4}+162 \pi^{6}-\pi^{8}+1224 \pi^{2} s-208 \pi^{4} s\right. \\
& \left.+8 \pi^{6} s-1296 s^{2}+288 \pi^{2} s^{2}-16 \pi^{4} s^{2}\right)
\end{aligned}
$$

and

$$
G_{3}^{\prime \prime}(t)=96(-3+\pi)(3+\pi)\left(-17 \pi^{2}+\pi^{4}+36 s-4 \pi^{2} s\right) .
$$

From the inequality

$$
\begin{aligned}
-17 \pi^{2}+\pi^{4}+\left(36-4 \pi^{2}\right) s & <-17 \pi^{2}+\pi^{4}+\left(36-4 \pi^{2}\right)\left(\frac{1}{4}\right) \\
& =9-18 \pi^{2}+\pi^{4} \\
& \cong-71.2438,
\end{aligned}
$$

$G_{3}^{\prime \prime}(s)<0$ and $G_{3}^{\prime}(s)$ is strictly decreasing for $1 / 4<s<\pi^{2} / 4$. Since $G_{3}^{\prime}(1 / 4)=12(-81+$ $\left.324 \pi^{2}-1574 \pi^{4}+164 \pi^{6}-\pi^{8}\right) \cong-24310.3, G_{3}^{\prime}(s)<0$ and $G_{3}(s)$ is strictly decreasing for $1 / 4<s<\pi^{2} / 4$. Therefore, we have $G_{1}(t)>G_{3}\left(\pi^{2} / 4\right)=576 \pi^{6}$ for $1 / 2<t<\pi / 2$. Next, the 
derivatives of $G_{4}(s)$ are

$$
\begin{aligned}
G_{4}^{\prime}(s)= & 4\left(-156 \pi^{4}+104 \pi^{6}-9 \pi^{8}+6240 \pi^{2} s-1344 \pi^{4} s+72 \pi^{6} s\right. \\
& \left.-14400 s^{2}+2880 \pi^{2} s^{2}-144 \pi^{4} s^{2}\right)
\end{aligned}
$$

and

$$
G_{4}^{\prime \prime}(s)=96\left(10-\pi^{2}\right)\left(26 \pi^{2}-3 \pi^{4}-120 s+12 \pi^{2} s\right) .
$$

From the inequality

$$
\begin{aligned}
26 \pi^{2}-3 \pi^{4}-120 s+12 \pi^{2} s & <26 \pi^{2}-3 \pi^{4}+\left(-120+12 \pi^{2}\right)\left(\frac{1}{4}\right) \\
& =-30+29 \pi^{2}-3 \pi^{4} \\
& \cong-36.0087
\end{aligned}
$$

$G_{4}^{\prime \prime}(s)<0$ and $G_{4}^{\prime}(s)$ is strictly decreasing for $1 / 4<s<\pi^{2} / 4$. Since $G_{4}^{\prime}(1 / 4)=4(-900+$ $\left.1740 \pi^{2}-501 \pi^{4}+122 \pi^{6}-9 \pi^{8}\right) \cong-2544.56, G_{4}^{\prime}(s)<0$ and $G_{4}(s)$ is strictly decreasing for $1 / 4<s<\pi^{2} / 4$. Therefore, we have $G_{2}(t)>G_{4}\left(\pi^{2} / 4\right)=48 \pi^{6}$ for $1 / 2<t<\pi / 2$. By the squeeze theorem, $F_{1}(t)>16$ for $1 / 2<t<\pi / 2$. Also, we have

$$
F_{1}(t)<\frac{G_{2}\left(\frac{1}{2}\right)}{3 \pi^{6}}
$$

for $1 / 2<t<\pi / 2$ and

$$
\begin{aligned}
F_{1}(0+)-\frac{G_{2}\left(\frac{1}{2}\right)}{3 \pi^{6}} & =\left(\pi^{2}-4\right)^{2}-16-\frac{G_{2}\left(\frac{1}{2}\right)}{3 \pi^{6}} \\
& =\left(\pi^{2}-4\right)^{2}-16-\frac{-300+840 \pi^{2}-327 \pi^{4}-163 \pi^{6}-5 \pi^{8}+3 \pi^{10}}{3 \pi^{6}} \\
& =\frac{300-840 \pi^{2}+327 \pi^{4}+163 \pi^{6}-19 \pi^{8}}{3 \pi^{6}} .
\end{aligned}
$$

By $300-840 \pi^{2}+327 \pi^{4}+163 \pi^{6}-19 \pi^{8} \cong 286.654$, we have

$$
F_{1}(0+)>\frac{G_{2}\left(\frac{1}{2}\right)}{3 \pi^{6}} .
$$

Thus, we can get $16<F_{1}(t)<F_{1}(0+)$ for $0<t<\pi / 2$. The proof of Theorem 2.1 is complete. 


\subsection{Proof of Theorem 2.2}

Proof of Theorem 2.2 We have

$$
\begin{aligned}
F_{1}(x) & =\frac{\pi^{2} x}{4+\sqrt{\left(\pi^{2}-4\right)^{2}+(2 \pi x)^{2}}}-\frac{8 x}{3+\sqrt{25+\frac{80}{3} x^{2}}} \\
& =\frac{x\left(-96+9 \pi^{2}+\sqrt{15} \pi^{2} \sqrt{15+16 x^{2}}-24 \sqrt{16-8 \pi^{2}+\pi^{4}+4 \pi^{2} x^{2}}\right)}{\left(9+\sqrt{15} \sqrt{15+16 x^{2}}\right)\left(4+\sqrt{16-8 \pi^{2}+\pi^{4}+4 \pi^{2} x^{2}}\right)} \\
& =\frac{x F_{2}(x)}{\left(9+\sqrt{15} \sqrt{15+16 x^{2}}\right)\left(4+\sqrt{16-8 \pi^{2}+\pi^{4}+4 \pi^{2} x^{2}}\right)} .
\end{aligned}
$$

The derivative of $F_{2}(x)$ is

$$
\begin{aligned}
F_{2}^{\prime}(x) & =\frac{16 \pi^{2} x\left(-6 \sqrt{15+16 x^{2}}+\sqrt{15} \sqrt{16-8 \pi^{2}+\pi^{4}+4 \pi^{2} x^{2}}\right)}{\sqrt{15+16 x^{2}} \sqrt{16-8 \pi^{2}+\pi^{4}+4 \pi^{2} x^{2}}} \\
& =\frac{16 \pi^{2} x F_{3}(x)}{\sqrt{15+16 x^{2}} \sqrt{16-8 \pi^{2}+\pi^{4}+4 \pi^{2} x^{2}}} .
\end{aligned}
$$

Here, we have $15\left(16-8 \pi^{2}+\pi^{4}+4 \pi^{2} x^{2}\right)-36\left(15+16 x^{2}\right)=3\left(-100-40 \pi^{2}+5 \pi^{4}-192 x^{2}+\right.$ $\left.20 \pi^{2} x^{2}\right)$. Since $-192+20 \pi^{2}>0$ and $-100-40 \pi^{2}+5 \pi^{4}-192 x^{2}+20 \pi^{2} x^{2}=0$ for $x=$ $\sqrt{\frac{100+40 \pi^{2}-5 \pi^{4}}{20 \pi^{2}-192}} \cong 1.198$, we have $F_{3}(x)<0$ for $0<x<\sqrt{\frac{100+40 \pi^{2}-5 \pi^{4}}{20 \pi^{2}-192}}$ and $F_{3}(x)>0$ for $x>$ $\sqrt{\frac{100+40 \pi^{2}-5 \pi^{4}}{20 \pi^{2}-192}}$. Therefore, $F_{2}(x)$ is strictly decreasing for $0<x<\sqrt{\frac{100+40 \pi^{2}-5 \pi^{4}}{20 \pi^{2}-192}}$ and strictly increasing for $x>\sqrt{\frac{100+40 \pi^{2}-5 \pi^{4}}{20 \pi^{2}-192}}$. From $F_{2}(0+)=0$ and

$$
\begin{aligned}
F_{2}(\alpha) & =-96+9 \pi^{2}+\sqrt{15} \pi^{2} \sqrt{15+16 \alpha^{2}}-24 \sqrt{16-8 \pi^{2}+\pi^{4}+4 \pi^{2} \alpha^{2}} \\
& =-96+9 \pi^{2}+\sqrt{15} \pi^{2}\left(\frac{\sqrt{15}\left(112-11 \pi^{2}\right)}{-48+5 \pi^{2}}\right)-24\left(\frac{192+32 \pi^{2}-5 \pi^{4}}{-48+5 \pi^{2}}\right) \\
& =0,
\end{aligned}
$$

we can get $F_{2}(x)>0$ for $x>\alpha$ and $\alpha$ is the best possible. The proof of Theorem 2.2 is complete.

\subsection{Proof of Theorem 2.3}

Proof of Theorem 2.3 We have

$$
\begin{aligned}
F_{1}(x) & =\frac{8 x}{3+\sqrt{25+\frac{256}{\pi^{2}} x^{2}}}-\frac{\pi^{2} x}{4+\sqrt{32+(2 \pi x)^{2}}} \\
& =\frac{\pi x\left(32-3 \pi^{2}-\pi \sqrt{25 \pi^{2}+256 x^{2}}+16 \sqrt{8+\pi^{2} x^{2}}\right)}{2\left(3 \pi+\sqrt{25 \pi^{2}+256 x^{2}}\right)\left(2+\sqrt{8+\pi^{2} x^{2}}\right)} \\
& =\frac{\pi x F_{2}(x)}{2\left(3 \pi+\sqrt{25 \pi^{2}+256 x^{2}}\right)\left(2+\sqrt{8+\pi^{2} x^{2}}\right)} .
\end{aligned}
$$


The derivative of $F_{2}(x)$ is

$$
\begin{aligned}
F_{2}^{\prime}(x) & =\frac{16 \pi x\left(\pi \sqrt{25 \pi^{2}+256 x^{2}}-16 \sqrt{8+\pi^{2} x^{2}}\right)}{\sqrt{25 \pi^{2}+256 x^{2}} \sqrt{8+\pi^{2} x^{2}}} \\
& =\frac{16 \pi x F_{3}(x)}{\sqrt{25 \pi^{2}+256 x^{2}} \sqrt{8+\pi^{2} x^{2}}} .
\end{aligned}
$$

Since $\pi^{2}\left(25 \pi^{2}+256 x^{2}\right)-16^{2}\left(8+\pi^{2} x^{2}\right)=-2048+25 \pi^{4} \cong 387.227$, we can get $\pi^{2}\left(25 \pi^{2}+\right.$ $\left.256 x^{2}\right)>16^{2}\left(8+\pi^{2} x^{2}\right)$ for $x>0$. Therefore, $F_{3}(x)>0$ and $F_{2}^{\prime}(x)>0$ for $x>0$. Since $F_{2}(x)$ is strictly increasing for $x>0$ and

$$
\begin{aligned}
F_{2}(\beta) & =32-3 \pi^{2}-\pi \sqrt{25 \pi^{2}+256 \beta^{2}}+16 \sqrt{8+\pi^{2} \beta^{2}} \\
& =32-3 \pi^{2}-\pi\left(\frac{512+96 \pi^{2}-17 \pi^{4}}{\pi\left(-32+3 \pi^{2}\right)}\right)+16\left(\frac{192-12 \pi^{2}-\pi^{4}}{2\left(-32+3 \pi^{2}\right)}\right) \\
& =0,
\end{aligned}
$$

we can get $F_{2}(x)>0$ for $x>\beta$ and $\beta$ is the best possible. The proof of Theorem 2.3 is complete.

\subsection{Proof of Theorem 2.4}

Lemma 2.6 For $x>0$, we have

$$
\frac{75600 \pi^{2} x}{\sqrt{8+\pi^{2} x^{2}}}+\frac{64 \pi^{2} x^{7}}{\sqrt{8+\pi^{2} x^{2}}}>\frac{25200 \sqrt{15} \pi^{2} x}{\sqrt{15+16 x^{2}}} .
$$

Proof We have

$$
\left(\frac{75600 \pi^{2} x}{\sqrt{8+\pi^{2} x^{2}}}+\frac{64 \pi^{2} x^{7}}{\sqrt{8+\pi^{2} x^{2}}}\right)^{2}-\left(\frac{25200 \sqrt{15} \pi^{2} x}{\sqrt{15+16 x^{2}}}\right)^{2}=\frac{256 \pi^{4} x^{2} F_{1}(x)}{\left(15+16 x^{2}\right)\left(8+\pi^{2} x^{2}\right)},
$$

where $F_{1}(x)=37209375+357210000 x^{2}-37209375 \pi^{2} x^{2}+567000 x^{6}+604800 x^{8}+$ $240 x^{12}+256 x^{14}$. Here, we have

$$
\begin{aligned}
F_{1}(x) & >37209375+357210000 x^{2}-37209375 \pi^{2} x^{2}+567000 x^{6} \\
& =70875\left(525+5040 x^{2}-525 \pi^{2} x^{2}+8 x^{6}\right) .
\end{aligned}
$$

We set $t=x^{2}$ and $F_{2}(t)=525+5040 t-525 \pi^{2} t+8 t^{3}$, then the derivative of $F_{2}(t)$ is $F_{2}^{\prime}(t)=$ $5040-525 \pi^{2}+24 t^{2}$. Since $F_{2}^{\prime}(t)=0$ for $t=\frac{1}{2} \sqrt{\frac{1}{2}\left(-1680+175 \pi^{2}\right)} \cong 2.4285$, we have $F_{2}^{\prime}(t)<$ 0 for $0<t<\frac{1}{2} \sqrt{\frac{1}{2}\left(-1680+175 \pi^{2}\right)}$ and $F_{2}^{\prime}(t)>0$ for $t>\frac{1}{2} \sqrt{\frac{1}{2}\left(-1680+175 \pi^{2}\right)}$. Hence,

$$
\begin{aligned}
F_{2}(t) & \geq F_{2}\left(\frac{1}{2} \sqrt{\frac{1}{2}\left(-1680+175 \pi^{2}\right)}\right) \\
& =\frac{35}{2}\left(30+48 \sqrt{70\left(-48+5 \pi^{2}\right)}-5 \pi^{2} \sqrt{70\left(-48+5 \pi^{2}\right)}\right) \\
& \cong 295.843
\end{aligned}
$$


for $t>0$. Therefore, $F_{1}(x)>0$ and the proof of Lemma 2.6 is complete.

Proof of Theorem 2.4 We have

$$
\begin{aligned}
F_{1}(x) & =\frac{8 x+\frac{32 x^{7}}{4725}}{3+\sqrt{25+\frac{80}{3} x^{2}}}-\frac{\pi^{2} x}{4+\sqrt{32+(2 \pi x)^{2}}} \\
& =\frac{x F_{2}(x)}{3150\left(9+\sqrt{15} \sqrt{15+16 x^{2}}\right)\left(2+\sqrt{8+\pi^{2} x^{2}}\right)},
\end{aligned}
$$

where $F_{2}(x)=151200-14175 \pi^{2}+128 x^{6}-1575 \sqrt{15} \pi^{2} \sqrt{15+16 x^{2}}+75600 \sqrt{8+\pi^{2} x^{2}}+$ $64 x^{6} \sqrt{8+\pi^{2} x^{2}}$. The derivative of $F_{2}(x)$ is

$$
\begin{aligned}
F_{2}^{\prime}(x) & =768 x^{5}-\frac{25200 \sqrt{15} \pi^{2} x}{\sqrt{15+16 x^{2}}}+\frac{75600 \pi^{2} x}{\sqrt{8+\pi^{2} x^{2}}}+\frac{64 \pi^{2} x^{7}}{\sqrt{8+\pi^{2} x^{2}}}+384 x^{5} \sqrt{\pi^{2} x^{2}+8} \\
& >-\frac{25200 \sqrt{15} \pi^{2} x}{\sqrt{15+16 x^{2}}}+\frac{75600 \pi^{2} x}{\sqrt{8+\pi^{2} x^{2}}}+\frac{64 \pi^{2} x^{7}}{\sqrt{8+\pi^{2} x^{2}}} .
\end{aligned}
$$

By Lemma 2.6, we have $F_{2}^{\prime}(x)>0$ and $F_{2}(x)$ is strictly increasing for $x>0$. From $F_{2}(0+)=$ $37800\left(4+4 \sqrt{2}-\pi^{2}\right) \cong-8041.96, F_{2}(\gamma)=0$ and $F(\infty)=\infty$, we can get $F_{2}(x)>0$ for $x>\gamma$. The proof of Theorem 2.4 is complete.

\section{Conclusions}

In this paper, we established some inequalities involving arctangent. The double inequality in Theorem 2.1 provides sharper quadratic estimations than (1.2) and (1.3) for a location away from zero. By Theorems 2.2, 2.3 and 2.4, we obtained Proposition 2.5 immediately.

\section{Competing interests}

The author declares that he has no competing interests.

\section{Acknowledgements}

I would like to thank referees for their careful reading of the manuscript and for their remarks and suggestions.

Received: 20 October 2016 Accepted: 30 January 2017 Published online: 08 February 2017

\section{References}

1. Shafer, RE: On quadratic approximation. SIAM J. Numer. Anal. 11(2), 447-460 (1974)

2. Shafer, RE: Analytic inequalities obtained by quadratic approximation. Publ. Elektroteh. Fak. Univ. Beogr., Ser. Mat. Fiz. 577-598, 96-97 (1977)

3. Shafer, RE: On quadratic approximation, II. Publ. Elektroteh. Fak. Univ. Beogr., Ser. Mat. Fiz. 602-633, 163-170 (1978)

4. Guo, B-N, Luo, Q-M, Qi, F: Sharpening and generalizations of Shafer-Fink's double inequality for the arc sine function. Filomat 27(2), 261-265 (2013)

5. Malešević, BJ: Application of $\lambda$-method on Shafer-Fink's inequality. Publ. Elektroteh. Fak. Univ. Beogr., Mat. 8, 103-105 (1997)

6. Malešević, BJ: An application of $\lambda$-method on inequalities of Shafer-Fink's type. Math. Inequal. Appl. 10(3), 529-534 (2007)

7. Nishizawa, Y: Sharpening of Jordan's type and Shafer-Fink's type inequalities with exponential approximations. Appl. Math. Comput. 269, 146-154 (2015)

8. Sun, J-L, Chen, C-P: Shafer-type inequalities for inverse trigonometric functions and Gauss lemniscate functions. J. Inequal. Appl. 2016, 212 (2016)

9. Zhu, L: On Shafer-Fink inequalities. Math. Inequal. Appl. 8(4), 571-574 (2005)

10. Zhu, L: On Shafer-Fink-type inequality. J. Inequal. Appl. 2007, 67430 (2007)

11. Zhu, L: New inequalities of Shafer-Fink type for arc hyperbolic sine. J. Inequal. Appl. 2008, 368275 (2008)

12. Zhu, L: On a quadratic estimate of Shafer. J. Math. Inequal. 2(4), 571-574 (2008)

13. Chen, C-P, Cheung, W-S: Sharp Cusa and Becker-Stark inequalities. J. Inequal. Appl. 2011, 136 (2011)

14. Chen, C-P, Sándor, J: Sharp inequalities for trigonometric and hyperbolic functions. J. Math. Inequal. 9(1), 203-217 (2015) 
15. Debnath, L, Mortici, C, Zhu, L: Refinements of Jordan-Steckin and Becker-Stark inequalities. Results Math. 67(1-2), 207-215 (2015)

16. Sun, Z-J, Zhu, L: Simple proofs of the Cusa-Huygens-type and Becker-Stark-type inequalities. J. Math. Inequal. 7(4), 563-567 (2013)

17. Zhu, L: Sharp Becker-Stark-type inequalities for Bessel functions. J. Inequal. Appl. 2010, 838740 (2010)

18. Zhu, L, Hua, J: Sharpening the Becker-Stark inequalities. J. Inequal. Appl. 2010, 931275 (2010)

19. Zhu, L: A refinement of the Becker-Stark inequalities. Math. Notes 93(3-4), 421-425 (2013)

Submit your manuscript to a SpringerOpen ${ }^{\circ}$ journal and benefit from:

- Convenient online submission

- Rigorous peer review

- Immediate publication on acceptance

- Open access: articles freely available online

- High visibility within the field

- Retaining the copyright to your article 